Int. J. Dev. Biol. 51: 173-176 (2007)

doi: $10.1387 / \mathrm{ijdb} .062221 \mathrm{tg}$

Developmental Expression Pattern

\title{
The expression of Fat-1 cadherin during chick limb development
}

\author{
TERENCE G. SMITH ${ }^{1, *}$, NICK VAN HATEREN ${ }^{2}$, CHERYLL TICKLE${ }^{1}$ and STUART A. WILSON ${ }^{2}$ \\ ${ }^{1}$ Division of Cell and Developmental Biology, University of Dundee, U.K. and ${ }^{2}$ Department of Molecular Biology and Biotechnology, \\ University of Sheffield, U.K.
}

\begin{abstract}
Cellular adhesion is fundamental to the behaviour of cell populations during embryonic development and serves to establish correct tissue pattern and architecture. The cadherin superfamily of cell adhesion proteins regulates cellular organization and additionally influences intracellular signalling cascades. Here we present for the first time a detailed account of chick Fat1 gene expression during embryogenesis visualised by whole-mount in situ hybridisation. In part, we focus on the expression pattern in limb buds that has not been accurately documented. While Fat-1 is generally expressed in epithelial tissues and its Drosophila counterpart Fat-like regulates formation of ectodermally-derived organs, in limb buds we have found that chick Fat-1 is uniquely restricted to mesenchyme. This Fat-1 expression pattern is remarkably dynamic throughout tissue differentiation, limb maturation and pattern formation. Diffuse expression of Fat-1 begins at stage $\mathrm{HH} 17$ as the limb bud is forming. It then becomes more proximal as the limb bud grows and is expressed within both tendon and muscle progenitors in the dorsal and ventral subectodermal mesenchyme. Later, Fat-1 transcripts were more abundant in anterior and posterior domains of the limb bud. During hand plate formation, Fat-1 transcripts were expressed in the mesenchyme adjacent to the wrist joint zone and in the interdigit mesenchyme.
\end{abstract}

KEY WORDS: Fat-1, cadherin, limb bud, cell adhesion, tendon

The cadherin superfamily consists of cell adhesion proteins that are highly conserved, contain multiple modular extracellular repeat motifs and homodimerise in a calcium-dependent manner (Tanoue and Takeichi, 2005). They constitute one of the many superfamilies of related genes that encode proteins that serve not only to tie cells together, but also to regulate intracellular signalling cascades. The Fat cadherins represent a distinct sub-group within the cadherin superfamily and are its largest members, generally containing 34 extracellular cadherin repeats, one or two laminin A-G motifs, 5 EGF domains and cytoplasmic EVH-1 and PDZ binding sites, giving them a molecular size of up to $600 \mathrm{kDa}$ (Tanoue and Takeichi, 2005). The Drosophila tumour suppressor Fat gene was discovered as a lethal mutation causing hyperplastic growth and altered cell-cell adhesion of larval imaginal discs, including those of the wing and leg (Bryant et al., 1988, Jaiswal et al., 2006). Studies have shown that Drosophila Fat genetically interacts with the wingless (wg) planar cell polarity pathway and in the eye contributes to dorsal-ventral patterning of ommatidia (Yang et al., 2002, Fanto and McNeill, 2004, Jaiswal et al., 2006).

There are 4 vertebrate Fat genes that closely resemble
Drosophila Fat (Tanoue and Takeichi, 2005). Recent analyses have indicated however that Fat 1-3are the true orthologues of the second Fat gene to be identified in Drosophila, Fat-like (Castillejo-Lopez et al., 2004, Rock et al., 2005, Tanoue and Takeichi, 2005). Fat 1-3 and Fat-like all encode proteins that contain a single laminin A-G motif, whereas the original Drosophila Fat has two of these motifs. A phylogenic tree shows that within this subfamily Fat-1 and Fat-3are closely related to each other, while Fat-2 is somewhat distinct (Tanoue and Takeichi, 2005). Fat-J is related to the original Drosophila Fatgene and is the most recently discovered fourth vertebrate ortholog (Hong et al., 2004). Where Drosophila Fat is ubiquitously expressed in some tissues, its ortholog Fat- $J$ is restricted to mesenchyme while Fat-1 is reciprocally expressed in epithelium (Rock et al., 2005). Thus, even though Fat-1 has diverged from Drosophila Fat, it seems to have taken over some of the

Abbreviations used in this paper: EGF, epidermal growth factor; PDZ, postsynaptic density protein, Drosophila disc large tumor suppressor and zo-1 protein; EVH-1, Ena/Vasp homology domain 1; wnt is the vertebrate homolog of wg (wingless).

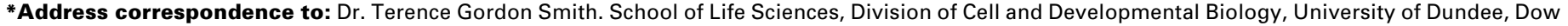
Street, Dundee, DD1 5EH, U.K. e-mail: tel_smith@yahoo.co.uk
} 


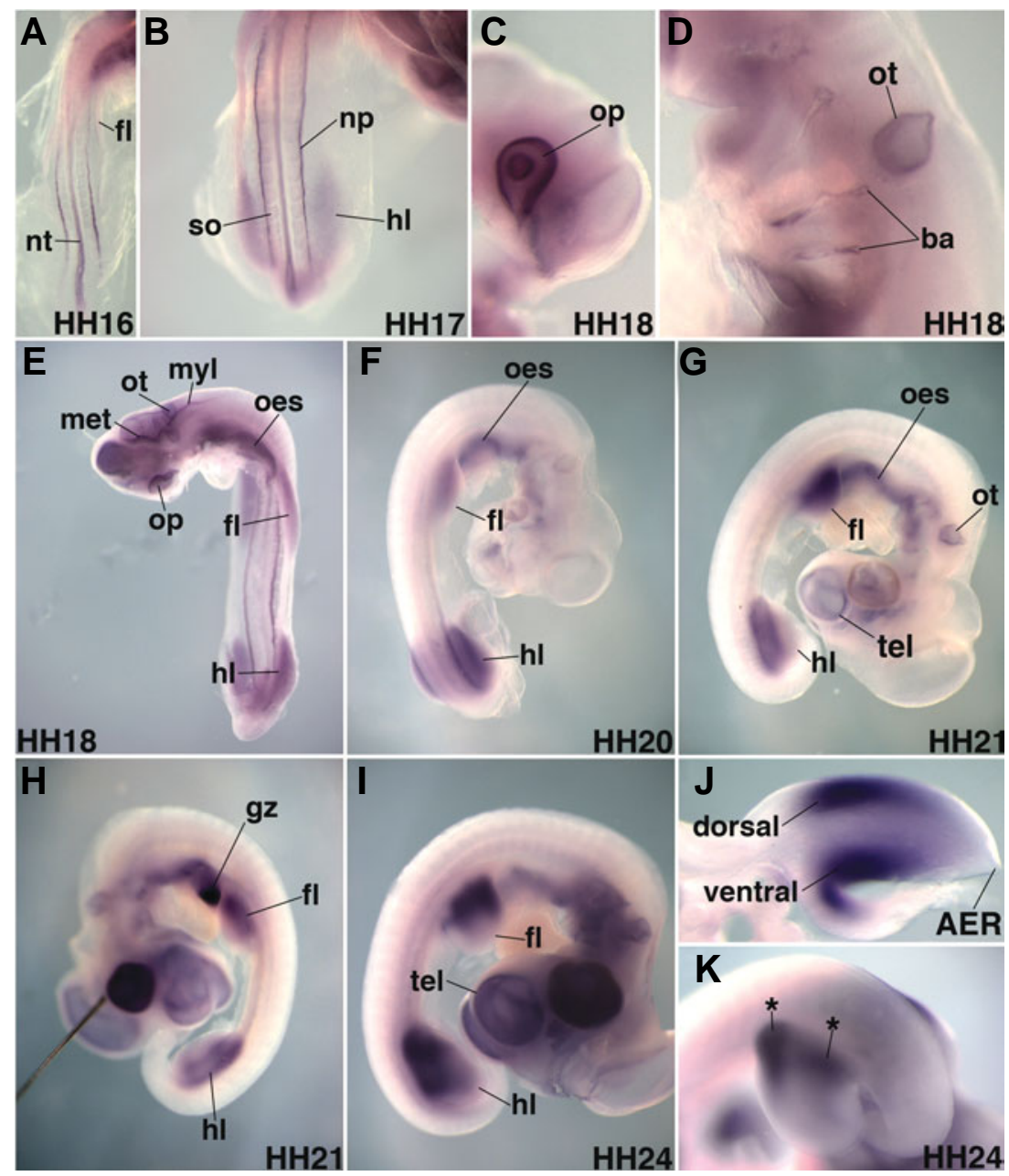

Fig. 1. Expression of Fat-1 during early limb bud formation. (A) Fat-1 expression in a stage HH16 chicken embryo. While expression is strong within the neural tube, it was not yet detected in the forming forelimb bud. (B) At $H H 17$, Fat-1 is present in the hindlimb and robustly expressed in the nephritic primordium. Very weak expression was detected in epithelial somites. (C) Fat-1 transcripts within the optic vesicle at HH18. (D) The epithelium lining the otic vesicle and branchial arches expressed Fat-1. (E) A ventral view of Fat-1 expression at stage HH18 in hindbrain, limb buds and oesophagus. (F) Limb bud expression at stage $\mathrm{HH} 20$ is just starting to become proximally restricted. (G) Transcription pattern in limb buds at HH21 is now more proximal, especially in the hindlimb bud. Fat-1 transcripts were detected within the telencephalon of the developing forebrain. (H) A lefthanded view of a HH21 embryo showing the prominent expression of Fat-1 in the presumptive gizzard. (I) Proximal Fat-1 expression in limb buds at HH24 and in lateral telencephalic vesicles. (J) Transverse section of a HH23 forelimb bud showing Fat-1 transcripts segregated into dorsal and ventral domains. These regions correspond to tendon and muscle primordia. (K) Close up view of a stage $\mathrm{HH} 24$ hindlimb showing the separation along the anteroposterior axis in the limb, marked by asterisks. Anterior is to the left, posterior the right. AER; apical ectodermal ridge. ba; branchial arches. fl; forelimb bud. gz; gizzard. hl; hindlimb bud. met; metencephalon. myl; myelencephalon. np; nephritic primordium. nt; neural tube. oes; oesophagus. op; optic vesicle. ot; otic vesicle. so; epithelial somites. tel; telencephalon.

Beginning at stage $\mathrm{HH} 16$, Fat-1 transcripts were expressed prominently in the neural tube, but were absent from the emerging forelimb bud (Fig. 1A). At $\mathrm{HH} 16$ and $\mathrm{HH} 17$, the nephritic primordium strongly expressed Fat-1 (Fig. 1A, B) and a diffuse expression now became apparent in

functions from the corresponding Fat ortholog. In fact, vertebrate Fat-1 is widely expressed in many epithelial tissues (Tanoue and Takeichi, 2005) and is necessary for the development of epithelial organs. For instance, deletion of Fat-1 in mice resulted in perinatal fatality due to lack of glomerular slit junction formation between the foot processes of podocytes in the developing kidney (Ciani et al., 2003). At lower penetrance, holoprosencephaly and anophthalmia resulted in Fat-1\% mutant embryos.

The expression of Fat-2transcripts and of Fat-2 protein have been detected within the cerebellum of rat embryos (Nakayama et al., 2002). Similarly, Fat-3is expressed in the rat spinal cord and central nervous system (Mitsui et al., 2002). In mouse, Fat$J$ is expressed in the neural tube, inner ear and intervertebral discs (Rock et al., 2005). Of all these Fat genes, Fat-1 shows the most complex and interesting expression pattern. However there are no current reports on the expression of Fat-1 in chick embryos. Furthermore, although the fact that Fat-1 expression in the mouse limb has been mentioned, the details of the expression pattern at different stages of development have not been described (Cox et al., 2000). Therefore, we have analysed chick Fat-1 expression throughout limb development and also report its expression in other regions of the chick embryo. the forelimb (not shown) and hindlimb (Fig. 1B). Epithelial somites displayed a very weak expression in both stages (Fig. 1A, B). Fat-1 transcripts were evident around the optic vesicle and lined the branchial arches (Fig. 1C, D, E). Persistent expression was maintained within the otic vesicle (Fig. 1D, E, G, Fig. 2H). We found Fat-1 transcripts expressed in the neuroepithelium lining the metacoele and myelocoele of the hindbrain and in the developing oesophagus (Fig. 1E, F, G).

During limb bud outgrowth, diffuse expression throughout the limb persisted at stage $\mathrm{HH} 18$ (Fig. 1E), which then became more proximal as development preceded through stage $\mathrm{HH} 20$ (Fig. 1F), HH21 (Fig. 1G) and HH24 (Fig. 1I, K). Cross section of a stage $\mathrm{HH} 23$ limb bud showed that Fat-1 transcripts had separated into two exclusive domains along the subectodermal mesenchyme (Fig. 1J). The similarity of the limb Fat-1 pattern in cross section to the expression of scleraxis, a marker of tendons (Schweitzer et al., 2001), suggests that tendon progenitors within the limb express Fat-1 cadherin. This region additionally contains migrating muscle progenitors (Schweitzer et al., 2001). The proximal restriction of Fat-1 in the early limb bud correlates well with the fact that only proximal and intermediate tendons expressed Fat-1 transcripts at later stages of development (Fig. 2A, B, C, D). Unlike scleraxis, Fat-1 expres- 
sion became stronger along the anterior and posterior edges of the limb bud, but weaker in the core (Fig. 1K).

At HH27, Fat-1 transcripts are seen as a V-shape in developing tendons of the stylopod and zeugopod (Fig. 2A, B, C, D). In contrast, the distal limb expression is not within tendons but is associated with forming cartilage elements. Likewise, the rat ortholog of Fat-1 is expressed in condensing cartilage in limbs (Ponassi et al., 1999). We noticed a progressive maturation of this distal Fat-1 expression pattern. As the forelimb developed, it initially contained a distal crescent-shaped domain of expression (Fig. 2A, B), which later disappeared and was replaced by two small areas at the joint interzones of the wrist-forming region (Fig. 2C). Finally, at $\mathrm{HH} 28$ the forelimb contained two long stripes of Fat-1 expression that extended along a narrow region between forming digits (Fig. 2D, E).

In mice, Fat-1\% mutant embryos have forebrain defects (Ciani et al., 2003) and throughout embryonic development we found prominent Fat-1 transcription in the proliferating neuroepithelium lining the lateral telencephalic vesicles (Fig. 1G, I, Fig. 2G). Further Fat-1 expression was noticed in the perineal area and anus (Fig. 2F). Lastly, we noted robust expression of Fat-1 in the presumptive gizzard, positioned on the left side of the body wall (Fig. 1H). Later, Fat-1 transcripts were still well maintained in the gizzard proper (Fig. 2I).

Expression of Fat-1 transcripts in rat, mouse and zebrafish are developmentally restricted to mainly epithelial tissues such as the eye, kidney, lung, gut, branchial arches, dermomyotome and neural tube and its Drosophila counterpart Fat-like regulates the formation of tubular organs of ectodermal origin (Ponassi et al., 1999, Cox et al., 2000, Castillejo-Lopez et al., 2004, Down et al., 2005). In contrast to this well-reported epithelial cell expression profile, mesenchymal cells within the limb bud expressed Fat-1 (Fig. 1J). The limb bud therefore represents a unique expression domain of the Fat-1 gene.

Fat-1 may have an important role in maintaining the separation of different cell lineages in limb bud mesenchyme, where no anatomical boundaries exist (Yajima et al., 2002). Rather, fixed expression domains of cadherins could group cell populations together through cellular adhesion to maintain the pattern of the limb bud. For example, while Fat-1 is proximally expressed (Fig. 1I, K), cadherin-11 transcripts are expressed distally (Kimura et al., 1995).

In Drosophila, Fat has multiple roles in the regulation of the conserved $\mathrm{wg} / \mathrm{wnt}$ signalling pathway during wing development (Jaiswal et al., 2006). It is known that $w g / w n t$ expression in the proximal Drosophilawing is regulated by a different mechanism than that in the distal wing (Rodriguez Dd Ddel et al., 2002). Somatic clones lacking Fat in proximal wing cells showed an increase in wg/wntexpression (Cho and Irvine, 2004, Jaiswal et al., 2006). Therefore, in the early chick limb bud the proximal restriction of Fat-1 (Fig. 1I, K) could inhibit wnt expression in those cells. Indeed, wnt-5a transcripts are excluded from the proximal limb bud cells at these stages (Loganathan et al., 2005)

Conversely in the distal Drosophila wing, Fat'- cells had no difference in $w g / w n t$ transcription but increased levels of cytoplasmic $\beta$-catenin/armadillo and therefore had higher levels of wg/wnt signalling (Jaiswal et al., 2006). We could therefore suppose that during digit formation in the chick distal limb, Fat1 may regulate wnt signalling, rather than wntexpression. In the distal limb bud at these stages, the expression pattern of wnt4 is similar to the pattern of Fat-1 in the wrist (Fig. 2C)
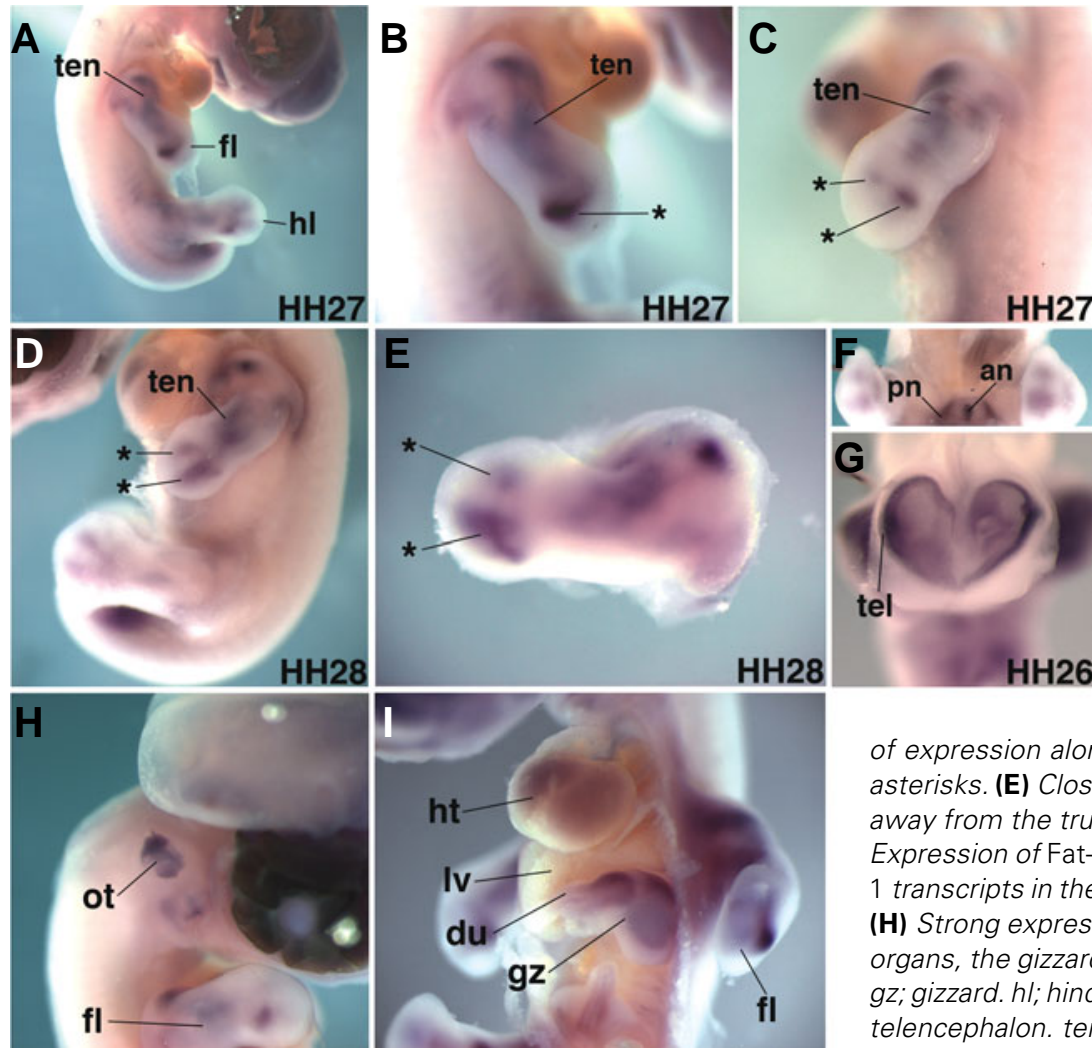

Fig. 2. Expression of Fat-1 during late limb formation. (A) HH27 embryo showing Fat-1 expression in fore- and hindlimbs. Note how the distal limb pattern differs between forelimb and hindlimb buds. The forelimb has a crescent-shaped domain, whereas the hindlimb contains a residual crescent domain overlapping two stripes of expression that extend along the interdigit mesenchyme. (B) Close up view of a stage HH27 forelimb showing the crescent domain marked by an asterisk. In the stylopod and zeugopod, Fat-1 transcripts are expressed by proximal and intermediate tendon progenitors in a $\mathrm{V}$-shape. (C) Close up view of a $\mathrm{HH} 27$ forelimb showing the transition of expression from crescent to two small marks, shown with asterisks. These marks delineate the interzone wrist-forming joint (D) At HH28 the forelimb has matured to the same stage as the hindlimb as both have extended stretches of expression along the interdigit mesenchyme, marked in the forelimb with asterisks. (E) Close view of a stage HH28 forelimb bud that has been dissected away from the trunk. Two asterisks mark the hand-plate Fat-1 expression. (F) Expression of Fat-1 in the perineal area and anus of the chick embryo. (G) Fat1 transcripts in the neuroepithelium of the left and right telencephalic vesicles. (H) Strong expression is seen in the developing otic vesicle. (I) Of the internal organs, the gizzard expressed Fat-1. an; anus. du; duodenum. fl; forelimb bud. gz; gizzard. hl; hindlimb bud. ht; heart. Iv; liver. ot; otic vesicle. pn; perineum. tel; telencephalon. ten; tendon primordia. 
(Loganathan et al., 2005). In addition, wnt-5a is expressed along the interdigit mesenchyme and both of these signals could be regulated by Fat-1 (Fig. 2D, E) (Loganathan et al., 2005).

However it must be taken into account that during evolution, the sequences encoding Drosophila Fat and its vertebrate homologues have diverged significantly, with alterations in key motifs such as the PDZ domain and in the arrangement of EGF and laminin A-G repeats (Ponassi et al., 1999, Rock et al., 2005). Thus, vertebrate Fat proteins may have acquired different cellular functions to those employed in Drosophila. It would be interesting to know how the properties conferred upon Fat proteins by their various protein domains could further influence pattern formation and gene expression. The chicken limb bud represents an ideal model system to test such properties (Tickle, 2003).

\section{Experimental Procedures}

\section{Fat-1 cadherin probe synthesis and in situ hybridisation}

The Fat-1 cadherin cDNA used to produce a DIG-labelled probe was obtained from the Chicken EST database, ChEST533a5 and sequence verified (Boardman et al., 2002). Not1 and T3 enzymes were used to linearize the plasmid and transcribe the RNA probe. In situhybridisation was performed as previously described (Smith et al., 2005), with a small modification. For stages $\mathrm{HH} 24-26$ and $\mathrm{HH} 27-28$, embryos were incubated with $10 \mu \mathrm{g} / \mathrm{ml}$ and $15 \mu \mathrm{g} / \mathrm{ml}$ respectively of Proteinase $\mathrm{K}$ at room temperature for 20 mins. Embryos were then post-fixed in 4\% PFA for a further $20 \mathrm{mins}$.

\section{Acknowledgements}

TGS was funded by The Royal Society and the MRC. NH, CT and SAW were funded by a joint BBSRC grant with $K$. Storey and $M$. Placzek.

\section{References}

BOARDMAN, P.E., SANZ-EZQUERRO, J., OVERTON, I.M., BURT, D.W., BOSCH, E., FONG, W.T., TICKLE, C., BROWN, W.R., WILSON, S.A. and HUBBARD, S.J. (2002). A comprehensive collection of chicken cdnas. Curr Biol 12: 1965-9.

BRYANT, P.J., HUETTNER, B., HELD, L.I., JR., RYERSE, J. and SZIDONYA, J (1988). Mutations at the fat locus interfere with cell proliferation control and epithelial morphogenesis in Drosophila. Dev Bio/129: 541-54.

CASTILLEJO-LOPEZ, C., ARIAS, W.M. and BAUMGARTNER, S. (2004). The fat-like gene of Drosophila is the true orthologue of vertebrate fat cadherins and is involved in the formation of tubular organs. J Biol Chem 279: 2403443.

CHO, E. and IRVINE, K.D. (2004). Action of fat, four-jointed, dachsous and dachs in distal-to-proximal wing signaling. Development 131: 4489-500.

CIANI, L., PATEL, A., ALLEN, N.D. and FFRENCH-CONSTANT, C. (2003). Mice lacking the giant protocadherin mfat 1 exhibit renal slit junction abnormalities and a partially penetrant cyclopia and anophthalmia phenotype. Mo/ Cel/ Bio/ 23: 3575-82.

COX, B., HADJANTONAKIS, A.K., COLLINS, J.E. and MAGEE, A.I. (2000). Cloning and expression throughout mouse development of mfat1, a homologue of the Drosophila tumour suppressor gene fat. Dev Dyn 217: 233-40.
DOWN, M., POWER, M., SMITH, S.I., RALSTON, K., SPANEVELLO, M., BURNS, G.F. and BOYD, A.W. (2005). Cloning and expression of the large zebrafish protocadherin gene, fat. Gene Expr Patterns 5: 483-90.

FANTO, M. and MCNEILL, H. (2004). Planar polarity from flies to vertebrates. $J$ Cell Sci 117: 527-33.

HONG, J.C., IVANOV, N.V., HODOR, P., XIA, M., WEI, N., BLEVINS, R., GERHOLD, D., BORODOVSKY, M. and LIU, Y. (2004). Identification of new human cadherin genes using a combination of protein motif search and gene finding methods. $J \mathrm{MO} / \mathrm{BiO} / 337$ : 307-17.

JAISWAL, M., AGRAWAL, N. and SINHA, P. (2006). Fat and wingless signaling oppositely regulate epithelial cell-cell adhesion and distal wing development in Drosophila. Development 133: 925-35.

KIMURA, Y., MATSUNAMI, H., INOUE, T., SHIMAMURA, K., UCHIDA, N., UENO, T., MIYAZAKI, T. and TAKEICHI, M. (1995). Cadherin-11 expressed in association with mesenchymal morphogenesis in the head, somite and limb bud of early mouse embryos. Dev Bio/169: 347-58.

LOGANATHAN, P.G., NIMMAGADDA, S., HUANG, R., SCAAL, M. and CHRIST, B. (2005). Comparative analysis of the expression patterns of wnts during chick limb development. Histochem Cel/ Bio/123: 195-201.

MITSUI, K., NAKAJIMA, D., OHARA, O. and NAKAYAMA, M. (2002). Mammalian fat3: A large protein that contains multiple cadherin and egf-like motifs. Biochem Biophys Res Commun 290: 1260-6.

NAKAYAMA, M., NAKAJIMA, D., YOSHIMURA, R., ENDO, Y. and OHARA, O. (2002). Megf1/fat2 proteins containing extraordinarily large extracellular domains are localized to thin parallel fibers of cerebellar granule cells. Mol Cell Neurosci 20: 563-78.

PONASSI, M., JACQUES, T.S., CIANI, L. and FFRENCH CONSTANT, C. (1999). Expression of the rat homologue of the Drosophila fat tumour suppressor gene. Mech Dev 80: 207-12.

ROCK, R., SCHRAUTH, S. and GESSLER, M. (2005). Expression of mouse dchs 1, fjx 1 and fat-j suggests conservation of the planar cell polarity pathway identified in Drosophila. Dev Dyn 234: 747-55.

RODRIGUEZ DD DDEL, A., TERRIENTE, J., GALINDO, M.I., COUSO, J.P. and DIAZ-BENJUMEA, F.J. (2002). Different mechanisms initiate and maintain wingless expression in the Drosophila wing hinge. Development 129: 3995 4004

SCHWEITZER, R., CHYUNG, J.H., MURTAUGH, L.C., BRENT, A.E., ROSEN, V., OLSON, E.N., LASSAR, A. and TABIN, C.J. (2001). Analysis of the tendon cell fate using scleraxis, a specific marker for tendons and ligaments. Development 128: 3855-66.

SMITH, T.G., SWEETMAN, D., PATTERSON, M., KEYSE, S.M. and MUNSTERBERG, A. (2005). Feedback interactions between mkp3 and erk map kinase control scleraxis expression and the specification of rib progenitors in the developing chick somite. Development 132: 1305-14.

TANOUE, T. and TAKEICHI, M. (2005). New insights into fat cadherins. J Cel/ Sci 118: $2347-53$

TICKLE, C. (2003). Patterning systems - from one end of the limb to the other. Dev Cel/ 4: 449-58.

YAJIMA, H., HARA, K., IDE, H. and TAMURA, K. (2002). Cell adhesiveness and affinity for limb pattern formation. Int J Dev Bio/46: 897-904.

YANG, C.H., AXELROD, J.D. and SIMON, M.A. (2002). Regulation of frizzled by fat-like cadherins during planar polarity signaling in the Drosophila compound eye. Ce//108: 675-88.

Received: 15th September 2006 Reviewed by Referees: 9th November 2006 Modified by Authors and Accepted for Publication: 20th December 2006 Published Online: 31st January 2007 